\title{
Preserving P-points in definable forcing
}

by

Jindřich Zapletal (Praha and Gainesville, FL)

\begin{abstract}
I isolate a simple condition that is equivalent to preservation of $\mathrm{P}$-points in definable proper forcing.
\end{abstract}

1. Introduction. Blass and Shelah [3], [2, Section 6.2] introduced the forcing property of preserving P-points. Here, a P-point is an ultrafilter $U$ on $\omega$ such that every countable subset of it has a pseudo-intersection in it: $\forall a_{n} \in U: n \in \omega \exists b \in U\left|b \backslash a_{n}\right|<\aleph_{0}$. While the existence of P-points is unprovable in $\mathrm{ZFC}$, they are plentiful under $\mathrm{ZFC}+\mathrm{CH}$. A forcing $P$ preserves an ultrafilter $U$ if every set $a \subset \omega$ in the extension either contains, or is disjoint from, a ground model element of the ultrafilter $U$; otherwise, $P$ destroys $U$. The forcing $P$ preserves P-points if it preserves all ultrafilters that happen to be P-points.

Several circumstances make this property a natural and useful tool. Every forcing adding a real number destroys some ultrafilter [2, Theorem 6.2.2]; if the forcing adds an unbounded real, then it destroys all non-P-point ultrafilters. A P-point, if preserved by a proper forcing, will again generate a P-point in the extension. Cohen and Solovay forcings both destroy all nonprincipal ultrafilters, and so preservation of P-points excludes the introduction of Cohen or random reals into the extension. Finally, preservation of P-points is itself preserved under the countable support iteration of proper forcing [3], [2, Theorem 6.2.6].

In the context of the theory of definable proper forcing [17], the preservation of P-points has two disadvantages: it trivializes when P-points do not exist (while the important properties of a definable forcing are typically independent of circumstances of this kind), and it refers to undefinable objects such as ultrafilters. As a result, it is not clear how difficult its verification property. 
might be, and what tools should be used for that verification. In this paper, I will resolve this situation by isolating a simple condition that is equivalent to the preservation of $\mathrm{P}$-points for definable proper forcing in the theory $\mathrm{ZFC}+\mathrm{LC}+\mathrm{CH}$. In order to state the theorem, I will need the following definitions.

Definition 1.1. A forcing $P$ does not add splitting reals if for every set $a \subset \omega$ in the extension there is an infinite ground model subset of $\omega$ which is either included in $a$ or disjoint from it.

This is a familiar property. Some forcings do not add splitting reals (Sacks forcing, the fat tree forcing [17, Section 4.4.3], the $E_{0}$ forcing [16], or Miller forcing [11], to include a diversity of examples), others do (most notably, Cohen and random forcing, as well as all the Maharam algebras [1], and with them all definable c.c.c. forcings adding a real). Clearly, a forcing adding a splitting real preserves no nonprincipal ultrafilters. I do not think that on its own not adding splitting reals is preserved under even two-step iteration. Its conjunction with the bounding property is preserved under the countable support iteration of definable forcings by [17, Corollary 6.3.8], and it is equivalent to the preservation of Ramsey ultrafilters by [17, Section 3.4].

Definition 1.2. A forcing $P$ has the weak Laver property if for every function $g \in \omega^{\omega}$ in the extension dominated by some ground model function there is a ground model infinite set $a \subset \omega$ and a ground model function $h: a \rightarrow \mathcal{P}(\omega)$ such that for every number $n \in a$, both $|h(n)|<2^{n}$ and $g(n) \in h(n)$ hold.

The weak Laver property is less well-known, and on the surface it appears to have nothing to do with preservation of any ultrafilters. It is a weakening of the more familiar Laver [2, Definition 6.3.27] or Sacks properties. Notably, it occurs in [2, Section 7.4.D] in parallel to the proof that the Blass-Shelah forcing preserves P-points. Some more complicated variants of it, iterable in the category of arbitrary proper forcings, appeared in [14, Section 7], to guarantee the preservation of certain more complicated properties of filters on $\omega$.

In order to precisely quantify the definability properties of the forcings involved, recall

Definition 1.3. A $\sigma$-ideal $I$ on a Polish space $X$ is universally Baire if for every universally Baire set $A \subset 2^{\omega} \times X$ the set $\left\{y \in 2^{\omega}: A_{x} \in I\right\}$ is universally Baire.

The class of universally Baire sets first appeared in [4]: these are the sets whose continuous preimages in Hausdorff spaces have the property of Baire. Suitable large cardinal assumptions imply that suitably definable subsets 
of Polish spaces are universally Baire [12], [8, Section 3.3], and analytic sets are universally Baire in ZFC. As [17] shows, a typical definable proper forcing adding a single real is of the form $P_{I}$ where $I$ is a universally Baire $\sigma$-ideal on a Polish space. The treatment of such a general class of forcings necessitates large cardinal assumptions at many ocassions. In order to prove ZFC theorems for a more restricted, but still significant, class of forcings, I will use the following definability notion considered for example by Sierpiński [7, Theorem 29.19]:

Definition 1.4. A $\sigma$-ideal $I$ on a Polish space $X$ is $\boldsymbol{\Pi}_{1}^{1}$ on $\boldsymbol{\Sigma}_{1}^{1}$ if for every analytic set $A \subset 2^{\omega} \times X$ the set $\left\{y \in 2^{\omega}: A_{y} \in I\right\}$ is coanalytic.

Most definable tree forcings are of the form $P_{I}$ for a $\Pi_{1}^{1}$ on $\Sigma_{1}^{1} \sigma$-ideal $I$. Now I am ready to state the main result of the paper. On the moral level, it says that in definable proper forcing, the preservation of P-points is equivalent to the conjunction of the weak Laver property and adding no splitting reals.

TheOREM 1.5. (CH) Suppose that $P$ is a proper forcing preserving $P$ points. Then $P$ has the weak Laver property and adds no splitting reals.

THEOREM 1.6. Suppose that there is a proper class of Woodin cardinals. If $I$ is a universally Baire $\sigma$-ideal on a Polish space such that the quotient forcing $P_{I}$ is proper, has the weak Laver property, and adds no splitting reals, then $P_{I}$ preserves $P$-points. If the ideal I is $\boldsymbol{\Pi}_{1}^{1}$ on $\boldsymbol{\Sigma}_{1}^{1}$ then the large cardinal assumption is not necessary.

The Continuum Hypothesis assumption in the former theorem is used only to ascertain the existence of many P-points. On the other hand, the definability assumption in the latter theorem is necessary:

ExAmple 1.7. (CH) There is a proper forcing which has the Laver property, adds no splitting reals, and fails to preserve a P-point.

The theorems can be used to swiftly argue that certain forcings preserve or do not preserve P-points. For example, the paper [15] shows that countable products of forcings of the form $P_{I}$, where $I$ is a $\sigma$-ideal generated by a compact collection of compact sets, do not add splitting reals. These products all have the weak Laver property, their associated ideal is $\boldsymbol{\Pi}_{1}^{1}$ on $\Sigma_{1}^{1}$ and therefore they must preserve P-points. A direct proof of this product preservation property seems to be out of reach. As another example, the forcings adding a bounded eventually different real must fail to have the weak Laver property, and so they never preserve P-points under $\mathrm{CH}$. On the other hand, the Blass-Shelah forcing of [2, Section 7.4.D] adds an unbounded eventually different real and still preserves P-points. 
The notation used in the paper follows the set-theoretic standard of [5]. The shorthand LC denotes the use of suitable large cardinal assumptions. If $A \subset X \times Y$ is a set and $x \in X$ is a point, then $A_{x}$ is the vertical section of the set $A$ corresponding to $x$.

2. Proof of Theorem 1.5. Suppose that the conclusion of Theorem 1.5 fails; I will argue that the assumption must fail as well. If $P$ adds a splitting real, then $P$ certainly destroys all nonprincipal ultrafilters. In the other case, the weak Laver property must fail for some function $f \in \omega^{\omega}$, and there is a condition $p \in P$ forcing that $\dot{g}<\check{f}$ is a counterexample. Let $U_{n}: n \in \omega$ be pairwise disjoint sets of the respective size $f(n)$, in some way identified with $f(n)$. Let $J$ be the ideal on the countable set $\operatorname{dom}(J)=\bigcup_{n} \mathcal{P}\left(U_{n}\right)$ generated by singletons and sets $a \subset \operatorname{dom}(J)$ such that for every number $n \in \omega$, either $a \cap \mathcal{P}\left(U_{n}\right)=0$ or $\left|\cap\left(a \cap \mathcal{P}\left(U_{n}\right)\right)\right|>2^{n}$, or $\left|U_{n} \backslash \bigcup\left(a \cap \mathcal{P}\left(U_{n}\right)\right)\right|>2^{n}$.

Claim 2.1. The ideal $J$ is an $F_{\sigma}$ proper ideal.

Proof. The set $F$ of generators is closed, and therefore compact, in the space $\mathcal{P}(\operatorname{dom}(J))$. The ideal generated by a closed set of generators is always $F_{\sigma}$, since the finite union map is continuous on the compact set $F^{n}$ for every $n \in \omega$, its image is again a compact set, and the ideal $J$ is the union of all of these countably many compact sets.

To see that $\operatorname{dom}(J) \notin J$, suppose that $a_{i}: i \in k$ are the generators of the ideal $J$. To show that they do not cover $\operatorname{dom}(J)$, find a number $n \in \omega$ such that $2^{n}>k$ and argue that there is a set $b \subset U_{n}$ not in any of the sets $a_{i}: i \in k$. First, partition $k$ into two pieces, $k=z_{0} \cup z_{1}$, such that for $i \in z_{0},\left|\cap\left(a_{i} \cap \mathcal{P}\left(U_{n}\right)\right)\right|>2^{n}$ holds, and for $i \in z_{1},\left|U_{n} \backslash \bigcup\left(a \cap \mathcal{P}\left(U_{n}\right)\right)\right|>2^{n}$ holds. Use a counting argument to find pairwise distinct elements $u_{i}: i \in k$ in the set $U_{n}$ so that for $i \in z_{0}, u_{i} \in \bigcap\left(a_{i} \cap \mathcal{P}\left(U_{n}\right)\right)$ holds, and for $i \in z_{1}$, $u_{i} \notin \bigcup\left(a \cap \mathcal{P}\left(U_{n}\right)\right)$ holds. The set $b=\left\{u_{i}: i \in z_{1}\right\}$ then belongs to none of the sets $a_{i}: i \in k$.

It follows from the definition of the ideal $J$ that the forcing $P$ below the condition $p$ adds a set $b \subset \operatorname{dom}(J)$ such that no ground model $J$-positive set can be disjoint from it, or included in it. Namely, consider the set $\dot{b}=\{c \subset$ $\left.U_{n}: \dot{g}(n) \in c, n \in \omega\right\}$. Suppose that $q \leq p$ is a condition, and $a \subset \operatorname{dom}(J)$ is a $J$-positive set. Then there must be infinitely many numbers $n \in \omega$ such that $a \cap \mathcal{P}\left(U_{n}\right) \neq 0$ and $\left|\bigcap\left(a \cap \mathcal{P}\left(U_{n}\right)\right)\right| \leq 2^{n}$; since $\dot{g}$ is forced by $p$ to be a counterexample to the weak Laver property, there must be a condition $r \leq q$ and a number $n \in \omega$ such that $r \Vdash \dot{g}(n) \notin \bigcap\left(\check{a} \cap \mathcal{P}\left(U_{n}\right)\right)$ and therefore $r \Vdash \check{a} \not \subset \dot{b}$. Similarly, there must be infinitely many numbers $n \in \omega$ such that $a \cap \mathcal{P}\left(U_{n}\right) \neq 0$ and $\left|U_{n} \backslash \bigcup\left(a \cap \mathcal{P}\left(U_{n}\right)\right)\right| \leq 2^{n}$, and by the failure of the weak Laver property, there must be a number $n$ and a condition $r \leq q$ forcing $\dot{g}(n) \in \bigcup\left(a \cap \mathcal{P}\left(U_{n}\right)\right)$ and so $\check{a} \cap \dot{b} \neq 0$. 
It is now enough to extend the ideal $J$ to a complement of a P-point, since then the previous paragraph shows that such a P-point cannot be preserved by the forcing $P$ below the condition $p$. Such an extension exists, since the ideal $J$ is $F_{\sigma}$; the construction is well-known, I am not certain to whom to attribute it, it certainly easily follows from some fairly old results.

Claim 2.2. ( $\mathrm{CH})$ Whenever $K$ is a proper $F_{\sigma}$ ideal on a countable set, there is a P-point ultrafilter disjoint from $K$.

Proof. By a result of [6], the quotient poset $\mathcal{P}(\omega) / I$ is countably saturated, in particular $\sigma$-closed. Any sufficiently generic filter over this poset will generate the desired P-point ultrafilter. Just build a modulo $K$ descending $\omega_{1}$-chain $a_{\alpha}: \alpha \in \omega_{1}$ of $K$-positive sets such that:

- $a_{\alpha+1}$ is either disjoint from or a subset of the $\alpha$ th subset of $\omega$ in some fixed enumeration;

- $a_{\alpha}$ is modulo finite included in all sets $a_{\beta}: \beta \in \alpha$ for every limit ordinal $\alpha$.

The first item shows that the sets $a_{\alpha}: \alpha \in \omega_{1}$ generate an ultrafilter disjoint from $K$, the second item is to ensure that this ultrafilter will be a $\mathrm{P}$-point. The induction itself is easy. At the successor step, note that if $b \subset \omega$ is the $\alpha$ th subset of $\omega$ in a given enumeration, then one of the sets $a_{\alpha} \cap b, a_{\alpha} \backslash b$ will be $K$-positive, and it will serve as $a_{\alpha+1}$. At the limit stage of induction, use the result of Mazur [10] to find a lower semicontinuous submeasure $\phi$ such that $K=\{b \subset \omega: \phi(b)<\infty\}$, enumerate $\alpha=\left\{\beta_{n}: n \in \omega\right\}$, and choose finite sets $b_{n} \subset \bigcap_{m \in n} a_{\beta_{m}}$ of $\phi$-mass $\geq n$. The set $a_{\alpha}=\bigcup_{n} b_{n}$ will work.

3. Proof of Theorem 1.6. This is more exciting. Assume that the assumptions hold. There are two auxiliary claims.

Claim 3.1. If $K$ is an $F_{\sigma}$ ideal on $\omega, p \in P$ is a condition, and $p \Vdash$ $\dot{b} \subset \omega$, then there are a ground model $K$-positive set and a condition $r \leq p$ forcing it to be either disjoint from, or a subset of, the set $\dot{b}$.

Proof. Use the result of Mazur [10] to find a lower semicontinuous submeasure $\phi$ on $\omega$ such that $J=\{c \subset \omega: \phi(c)<\infty\}$. Find pairwise disjoint sets $c_{n} \subset \omega$ such that $\phi\left(c_{n}\right)>n \cdot 2^{2^{n}}$, this for every $n \in \omega$. Use the weak Laver property to find an infinite set $a \subset \omega$, sets $d_{n} \subset \mathcal{P}\left(c_{n}\right)$ of the respective size $\leq 2^{n}$, and a condition $q \leq p$ such that $q \Vdash \forall n \in \check{a} \dot{b} \cap \check{c}_{n} \in \check{d}_{n}$. Use the subadditivity of the submeasure $\phi$ to find sets $e_{n} \subset c_{n}$ of submeasure $\geq n$ such that $\forall f \in d_{n} f \cap e_{n}=0 \vee e_{n} \subset f$, this for every $n \in a$. Thus $q \Vdash \forall n \in a \check{e}_{n} \subset \dot{b} \vee \check{e}_{n} \cap \dot{b}=0$. Since $P$ adds no splitting reals, there is a condition $r \leq q$ and an infinite subset $a^{\prime} \subset a$ such that $r \Vdash \forall n \in a^{\prime} \check{e}_{n} \subset \dot{b} \vee \forall n \in a^{\prime} \check{e}_{n} \cap \dot{b}=0$. In the first case, the ground model 
$J$-positive set $\bigcup_{n \in a^{\prime}} e_{n}$ is forced to be a subset of $\dot{b}$, in the other case, this set is forced to be disjoint from $\dot{b}$ as desired.

Claim 3.2. (ZFC $+\mathrm{LC)}$ If $U$ is a P-point and $J$ is a universally Baire ideal disjoint from $U$, then there is an $F_{\sigma}$ ideal $K \supset J$ disjoint from $U$. If $J$ is analytic then no large cardinals are needed.

Note that Claims 2.2 and 3.2 together yield a complete characterization of analytic ideals on $\omega$ that are disjoint from a P-point under $\mathrm{CH}$ : these are exactly those ideals that can be extended to nontrivial $F_{\sigma}$ ideals.

Proof. I will prove the large cardinal version with a direct determinacy argument and then use the Kechris-Louveau-Woodin dichotomy to argue for the analytic case in ZFC.

Recall the Galvin-Shelah game theoretic characterization of P-points: the ultrafilter $U$ is a P-point if and only if Player I has no winning strategy in the P-point game where he chooses sets $a_{n} \in U$, Player II chooses their finite subsets $b_{n} \subset a_{n}$, and Player II wins if $\bigcup_{n} b_{n} \in U$ [2, Theorem 4.4.4]. Now consider the same game, except the winning condition for Player II is replaced with $\bigcup_{n} b_{n} \notin J$. This is certainly easier to win for Player II, and so Player I still does not have a winning strategy. Now, however, the payoff set is universally Baire and one can use the large cardinal assumptions and determinacy results [9] to argue that the game is determined and Player II must have a winning strategy $\sigma$.

Let $M$ be a countable elementary submodel of a large enough structure containing the strategy $\sigma$. For every position $p \in M$ of the game that respects the strategy $\sigma$ and ends with a move of Player II, let $u_{p}=\{b \in$ $[\omega]^{<\aleph_{0}}: \exists a \in U p^{\curlyvee} a^{\frown} b$ is a position respecting the strategy $\left.\sigma\right\}$ and let $F_{p}=\left\{c \subset \omega: c\right.$ has no subset in $\left.u_{p}\right\}$. The sets $F_{p} \subset \mathcal{P}(\omega)$ are closed and disjoint from the ultrafilter $U$, since for every set $a \in U$ the strategy $\sigma$ must answer $a$ with its subset. Thus, the sets $F_{p}: p \in M$ generate an $F_{\sigma}$ ideal $K$ on $\omega$ disjoint from the ultrafilter $U$. I must show that $J \subset K$ holds.

Suppose $c \subset \omega$ is not in the ideal $K$. By induction on $n \in \omega$ find sets $a_{n} \in U \cap M$ such that when Player I plays these sets in succession, the strategy $\sigma$ always responds with a subset of $c$. Suppose the sets $a_{n}: n \in m$ have been built, and let $p \in M$ be the corresponding position of the game. Since $c \notin F_{p}$, there must be a set $a_{m}$ such that the strategy responds to the move $a_{m}$ by a subset of $c$. This concludes the inductive construction. In the end, the strategy $\sigma$ won the infinite play against the sequence $a_{n}: n \in \omega$ of Player I's challenges. Thus the set $\bigcup_{n} b_{n}$ it produced was not $J$-positive. This set is a subset of the set $c$ by the inductive construction, and therefore $c \notin J$ as required. 
Now for the ZFC case, let $J$ be an analytic ideal disjoint from the P-point ultrafilter $U$. If $J$ can be separated from $U$ by an $F_{\sigma}$ set $K_{0}$, then the ideal $K$ generated by this set is still $F_{\sigma}$, still disjoint from $U$, and it includes $J$ as desired. If $J$ cannot be so separated, then the Kechris-Louveau-Woodin dichotomy [7, Theorem 21.22] shows that there is a perfect set $C \subset J \cap U$ such that $C \cap U$ is countable and dense in $C$. I will use it to construct a winning strategy for Player I in the P-point game, yielding a contradiction and completing the proof. Let $c_{n}: n \in \omega$ be an enumeration of the set $C \cap U$. Player I will win by playing sets $a_{n} \in C \cap U$ and on the side writing down finite initial segments $b_{n}^{\prime} \subset a_{n}$ which include Player II's answer $b_{n}$ in such a way that

- $a_{n}$ contains $\bigcup_{i \in n} b_{i}^{\prime}$ as an initial segment;

- $a_{n} \neq c_{n}$ and $c_{n}$ does not contain $\bigcup_{i \in n+1} b_{i}^{\prime}$ as an initial segment.

This is easily possible. In the end, the set $\bigcup_{n \in \omega} b_{n}^{\prime} \subset \omega$ is the limit of the sets $a_{n} \in C \cap U$, and therefore it belongs to $C$ by the first item, and it is not equal to any of the sets in $C \cap U$ by the second item. Consequently, it must belong to the ideal $J$, and since the set $\bigcup_{n \in \omega} b_{n}$ is included in it, it means that Player I won.

Theorem 1.6 now follows easily. Suppose $P$ is a proper forcing, $P=P_{I}$ for some universally Baire $\sigma$-ideal on a Polish space $X, U$ is a P-point, $B \in P_{I}$ is a condition and $B \Vdash \dot{b} \subset \omega$ is a set. I must find a condition $C \subset B$ and a set $a \in U$ such that $C \Vdash \dot{b} \cap \check{a}=0 \vee \check{a} \subset \dot{b}$. By strengthening the condition $B$ I may assume that there is a Borel function $f: B \rightarrow \mathcal{P}(\omega)$ such that $B \Vdash \dot{b}=\dot{f}\left(\dot{x}_{\text {gen }}\right)$. Consider the set $J_{0}=\{a \subset \omega: \exists C \subset B C \Vdash \check{a} \cap \dot{b}=0 \vee C \Vdash$ $\check{a} \subset \dot{b}\}=\{a \subset \omega:\{x \in B: f(x) \cap a=0\} \notin I \vee\{x \in B a \subset f(x)\} \notin I\}$. If it is not disjoint from the P-point $U$, then we are done. If $J_{0} \cap U=0$, then even the ideal $J$ generated by $J_{0}$ is disjoint from $U$. The ideal $J$ is universally Baire, and if the $\sigma$-ideal $I$ is $\boldsymbol{\Pi}_{1}^{1}$ on $\boldsymbol{\Sigma}_{1}^{1}$ then $J$ is in fact analytic. Claim 3.2 now shows that there is an $F_{\sigma}$ ideal $K \supset J$ disjoint from $U$. Claim 3.1 shows that there is a condition $C \subset B$ and a $K$-positive set $a \subset \omega$ such that $C \Vdash \check{a} \cap \dot{b}=0$ or $C \Vdash \check{a} \subset \dot{b}$. This however contradicts the definition of the set $J_{0} \subset K$ !

4. Proof of Example 1.7. Suppose that the Continuum Hypothesis holds, and fix a Ramsey ultrafilter $U$. Consider the partial order $P_{U}$ consisting of those pruned trees $T \subset 2^{<\omega}$ such that there is a set $a \in U$ such that a node in $T$ is a split node if and only if its length is in the set $a$ ordered by inclusion. The forcing $P_{U}$ witnesses the conclusion of Example 1.7. It is clear that the generic real $\dot{x}_{\text {gen }}$, the union of the intersection of all trees in the generic filter, is a function in $2^{\omega}$ which is not constant on any set in the ultrafilter $U$. The forcing also has the Sacks property and adds no splitting reals. 
Instead of the somewhat slippery argument for this latter statement, I will prove a closely related fact. Consider the symmetric Sacks forcing $P$ of [13]. It consists of those pruned trees $T \subset 2^{<\omega}$ such that there is an infinite set $a \subset \omega$ such that a node in $T$ is a splitnode if and only if its length is in the set $a$, ordered by inclusion. It is not difficult to see that the forcing $P$ splits into a two-step iteration, $P=Q * P_{\dot{U}}$, where $Q$ is the ordering of infinite subsets of $\omega$ with modulo finite inclusion, and $\dot{U}$ is the $Q$-name for the Ramsey ultrafilter added by $Q$. A standard fusion argument directly transferred from the usual Sacks forcing case shows that the symmetric Sacks forcing has the Sacks property. It is significantly harder to show that $P$ adds no splitting reals; it follows for example from the upcoming work of [15]. Now, summing up, it is clear that in the $Q$ extension, there is a forcing, namely $P_{U}$, which has the Sacks property and adds no splitting reals, and adds a function from $\omega$ to 2 which is not constant on any set in the Ramsey ultrafilter $U$.

5. Applications of the main theorems. Theorems 1.5 and 1.6 can be used in two directions: to ensure that certain forcings preserve P-points, and to prove that other forcings do not preserve P-points. In this brief section I will give examples of both.

An important and well studied class of forcings consists of the quotient forcings obtained from ideals on a Polish space $X$ generated by a compact collection of compact sets in the hyperspace $K(X)$ [17, Theorem 4.1.8]; this is a slight generalization of the fairly common limsup infinity tree forcings of [14]. These quotient forcings do not add splitting reals and have the weak Laver property; therefore, they preserve P-points. Their countable products are more difficult to analyze. However, a simple fusion argument shows that the products possess the weak Laver property, and a subtle combinatorial argument [15] shows that the products do not add splitting reals. Theorem 1.6 then implies the conclusion:

Proposition 5.1. The countable product of quotient forcings of $\sigma$-ideals generated by a compact collection of compact sets preserves $P$-points.

The methods of [15] show that many other forcings, including the wide Silver forcing, symmetric Sacks forcing [13], and the $E_{0}$ and $E_{2}$ forcings [17, Section 4.7], do not add splitting reals. The forcings just named all have the weak Laver property, and therefore, by Theorem 1.6, they also preserve $\mathrm{P}$-points. This is perhaps not quite surprising, but a direct proof seems to be out of reach.

As an example of the application in the opposite direction, let me include

Proposition 5.2. (CH) If $P$ is a forcing adding a bounded eventually different real, then $P$ fails to preserve P-points. 
Note that every bounding forcing making the set of all ground model reals meager falls into this category essentially by [2, Theorem 2.4.7]. Thus, for example, forcing with an ideal associated with a Ramsey capacity is bounding and adds no splitting reals [17, Theorem 4.3.25], but it must destroy a P-point. On the other hand, the Blass-Shelah forcing makes the set of ground model reals meager, it is not bounding, and it preserves P-points.

Proof. It will be enough to show that $P$ fails the weak Laver property. Suppose $\dot{g}$ and $f$ are a $P$-name and a function in $\omega^{\omega}$ respectively such that $P \Vdash \dot{g}<\check{f}$ and for every ground model function $h \in \omega^{\omega}, \dot{g} \cap \check{h}$ is finite. Let $\omega=\bigcup_{n} b_{n}$ be a partition of $\omega$ into finite sets of the respective size $2^{n}$, let $\bar{f}(n)$ be the set $\pi_{i \in b_{n}} f(i)$ and let $\bar{g} \in \Pi_{n} \bar{f}(n)$ be the name for the function in the extension defined by $\bar{g}(n)=\dot{g} \mid \breve{b}(n)$. I claim that $\bar{f}, \bar{g}$ witness the failure of the weak Laver property.

Indeed, if $a \subset \omega$ were an infinite set, $h$ a ground model function on $a$ such that $h(n)$ is a subset of $\bar{f}(n)$ of size $<2^{n}$ and $p \in P$ a condition forcing $\forall n \in a \bar{g}(n) \in \check{h}(n)$, one could find surjections $u_{n}: b_{n} \rightarrow h(n)$ for every number $n \in a$, find a function $k \in \omega^{\omega}$ such that $k(i)=u_{n}(i)(i)$ for every $n \in a$ and every $i \in b_{n}$, and conclude that $p \Vdash \check{k} \cap \dot{g}$ is infinite. This contradicts the assumptions on the name $\dot{g}$.

Acknowledgements. This research was partially supported by NSF grant DMS 0801114 and Institutional Research Plan No. AV0Z10190503 and grant IAA100190902 of GA AV ČR.

\section{References}

[1] B. Balcar, T. Jech, and T. Pazák, Complete ccc algebras, the order sequential topology, and a problem of von Neumann, Bull. London Math. Soc. 37 (2005), 885-898.

[2] T. Bartoszyński and H. Judah, Set Theory. On the Structure of the Real Line, A K Peters, Wellesley, MA, 1995.

[3] A. Blass and S. Shelah, There may be simple $p_{\aleph_{1}}$ - and $p_{\aleph_{2}}$-points and the RudinKeisler ordering may be downward directed, Ann. Pure Appl. Logic 33 (1987), 213243.

[4] Q. Feng, M. Magidor, and H. Woodin, Universally Baire sets of reals, in: H. Judah et al. (eds.), Set Theory of the Continuum, MSRI Publ. 26, Springer, New York, 1992, 203-242.

[5] T. Jech, Set Theory, Academic Press, San Diego, 1978.

[6] W. Just and A. Krawczyk, On certain Boolean algebras $\mathcal{P}(\omega) / I$, Trans. Amer. Math. Soc. 285 (1984), 411-429.

[7] A. S. Kechris, Classical Descriptive Set Theory, Springer, New York, 1994.

[8] P. B. Larson, The Stationary Tower Forcing, Univ. Lecture Ser. 32, Amer. Math. Soc., Providence, RI, 2004.

[9] A. Martin, An extension of Borel determinacy, Ann. Pure Appl. Logic 49 (1990), 279-293. 
[10] K. Mazur, $F_{\sigma}$-ideals and $\omega_{1} \omega_{1}^{*}$-gaps in the Boolean algebra $P(\omega) / I$, Fund. Math. 138 (1991), 103-111.

[11] A. Miller, Rational perfect set forcing, in: Axiomatic Set Theory, Contemp. Math. 31, Amer. Math. Soc., Providence, 1983, 143-159.

[12] I. Neeman and J. Zapletal, Proper forcing and absoluteness in $L(R)$, Comment. Math. Univ. Carolin. 39 (1998), 281-301.

[13] A. Rosłanowski, n-localization property, J. Symbolic Logic 71 (2006), 881-902.

[14] A. Rosłanowski and S. Shelah, Norms on possibilities I: forcing with trees and creatures, Mem. Amer. Math. Soc. 141 (1999), no. 671.

[15] S. Shelah and J. Zapletal, Products of finite sets with submeasures, in preparation, 2009.

[16] J. Zapletal, Parametrized Ramsey theorems and proper forcing, submitted, 2007.

[17] —, Forcing Idealized, Cambridge Tracts in Math. 174, Cambridge Univ. Press, Cambridge, 2008.

Institute of Mathematics of the AS CR

Žitná 25

CZ-115 67 Praha 1, Czech Republic

E-mail: zapletal@math.cas.cz
Department of Mathematics

University of Florida Gainesville, FL 32611-8105, U.S.A.

Received 28 February 2008;

in revised form 22 April 2009 